\title{
Engagement y trayectoria profesional en técnicos de deporte adaptado
}

\author{
Engagement and career path in adapted sports coaches
}

\section{Engajamento e carreira profissional no esporte adaptado}

\author{
Silvia Barnet, Jordi Segura Bernal, Josep Oriol Martínez-Ferrer y Myriam Guerra Balic \\ Facultat de Psicologia, Ciències de l'Educació i de l'Esport Blanquerna - Universitat Ramon Llull
}

\begin{abstract}
Resumen: El presente estudio abre futuras líneas de investigación en el ámbito de la psicología positiva en el deporte. Se realizó un análisis descriptivo de la trayectoria del técnico de deporte adaptado y se profundizó en conocer su nivel de Engagement y las tres dimensiones que conforman este constructo. Posteriormente se realizó un análisis comparativo con técnicos de deporte general. En el estudio participaron 45 técnicos de deporte adaptado y 45 técnicos de deporte general, seleccionados de forma no probabilística e intencional. Para la medición del Engagement se utilizó el cuestionario Engagement: Utrecht Work Engagement Scale (Schaufeli y Bakker, 2003); y para profundizar en el perfil del técnico, un breve cuestionario sobre la trayectoria profesional de los técnicos de deporte, a partir del modelo de Pérez-Ramírez (2002b). Los resultados muestran que los participantes presentan altos niveles de Engagement y se identifican elementos comunes entre los técnicos de deporte adaptado y el resto de técnicos. Más de la mitad del grupo estudiado han sido deportistas de competición, no se dedican exclusivamente a entrenar y presentan un perfil más polideportivo.

Palabras clave: Compromiso, carrera profesional, entrenadores, deportistas con discapacidad.

Abstract: The current study opens future lines of research in the field of positive Psychology in Sports. A descriptive analysis of the adapted sports coach career path was conducted, going in depth into their level of Engagement and the three dimensions that make up this construct. Later, a comparative analyses with general sports coaches was completed. The study was developed with a sample of 45 adapted sports coaches and 45 general sports coaches. The Engagement: Utrecht Work Engagement Scale (Schaufeli y Bakker, 2003) was used to measure de level of Engagement, and a
\end{abstract}

brief questionnaire drawn on that from Pérez-Ramirez (2002b) about the career path of sports coaches was applied to explore the coaches' profile. The participants show high levels of Engagement and common elements are identified among both groups. More than a half of the group have been competitive athletes, do not work exclusively as a coach and have a multisport profile.

Key words: Engagement, professional career, coaches, athletes with disabilities.

Resumo: O presente estudo abre futuras linhas de investigação no âmbito da psicologia positiva no desporto. Realizou-se uma análise descritiva da trajetória do técnico do desporto adaptado e estudou-se a fundo o seu nível de Engagement e as três dimensóes que definem este constructo.Posteriormente, realizou-se uma análise comparativa com técnicos do desporto em geral. $\mathrm{O}$ estudo foi realizado com uma amostra de 45 técnicos de desporto adaptado e 45 técnicos de desporto geral, selecionados de forma náo probabilística e intencional. Para a medição de Engagementutilizou-se o inquérito Engagement: Utrecht Work EngagementScale (Schaufeli y Bakker, 2003); e para estudar a fundo o perfil do técnico, um breve inquérito sobre a trajetória profissional dos técnicos do desporto, a partir do modelo PérezRamirez (2002b). Os resultados mostram que os participantes apresentam altos níveis de Engagement e identificam-se elementos comuns entre os técnicos de desporto adaptado e o resto de técnicos. Mais de metade do grupo estudado foi desportista de competição, não se dedica exclusivamente a treinar e apresenta um perfil mais polidesportivo.

Palavra chave: Engagement, carreiraprofissional, treinadores, atletas comdeficiência.

\section{Introducción}

El estudio aborda la actuación del técnico de deporte adaptado desde el enfoque de la Psicología Positiva (Seligman, Steen, Park y Peterson, 2005), un modelo que tiene como finalidad estudiar el bienestar y las fortalezas del ser humano, integrando conocimientos de los paradigmas conductual-cognitivo y humanista. La Psicología Positiva permite analizar el desarrollo de competencias, estrategias y herramientas para trabajar con el potencial y los puntos fuertes del individuo (pensamiento positivo) y pone atención en la mejora de la calidad de vida, la promoción de la salud y el bienestar (Vera, 2006).

Después de la segunda Guerra Mundial se empezó a desarrollar una nueva dimensión del deporte y del deporte

Dirección para correspondencia [Correspodence address]: Silvia Barnet Lopez. Universitat Ramón Llull. C/ Císter, 34. Barcelona (España). E-mail:SilviaBL0@blanquerna.url.edu adaptado, relacionándolos con el nuevo concepto de salud. En 1944 el Dr. L. Guttmann, como director del Centro de Stoke-Mandeville (Gran Bretańa), desarrolló nuevas aportaciones en cuanto a técnicas de actividad física y deportiva de rehabilitación para personas con discapacidades físicas. Posteriormente, en 1948 (coincidiendo con la celebración de los Juegos Olímpicos de Londres) Guttmann organizó la primera competición reconocida de deporte adaptado para esta población aunque existían otros precedentes. Los Juegos de Stoke-Mandeville se convirtieron en un referente en la historia del deporte adaptado, sentando así las bases del deporte paralímpico actual (Guttmann, 1976).

Con la construcción del "estado de bienestar", tras las dos grandes guerras mundiales del siglo XX, los países industrializados emprenden una readaptación de costumbres 
y valores y aumenta la disposición a mejorar la calidad de vida de las personas con discapacidad, con fuerte implicación de profesionales de la medicina, la psicología y la sociología. Desde esas disciplinas se promueve una aproximación de la actividad física y el deporte a la población de personas con discapacidad; entendida, primero, como un buen método de rehabilitación y, posteriormente como elemento de integración y normalización social, dando lugar a la actividad física adaptada y al deporte adaptado (Martínez-Ferrer, 2008).

El concepto de discapacidad también ha ido evolucionando. Según la Organización Mundial de la Salud (OMS, 1980), el término discapacidad se refiere a toda restricción o ausencia de la capacidad para realizar una actividad, en la forma o dentro del margen que se considera normal para un ser humano. La definición fue modificada en el año 2000 con el objetivo de ofrecer una clasificación con un lenguaje unificado y estandarizado, relacionado con el ámbito de la salud, tal y como refiere el plan integral para la actividad física y el deporte presentado en España (CSD, 2009). La Clasificación Internacional del Funcionamiento, de la Discapacidad y de la Salud (C.I.F) habla de discapacidad como el resultado de la interacción entre la condición de salud de la persona y sus factores personales, y los factores contextuales que pueden llegar a ser facilitadores o limitadores del desarrollo de una determinada actividad o del desenvolvimiento personal en forma de participación (Reina, 2014). Los expertos señalan que en los últimos años la investigación en relación a los diferentes ámbitos del deporte ha ido en aumento, aunque en el ámbito de la investigación aplicada a la AFA ha disminuido (Pérez-Tejero, 2009).

El deporte adaptado en su conjunto es más complejo que el deporte general. Esto se debe, en primer lugar, a la diversidad de perfiles de los deportistas a causa de los tipos y grados de discapacidad, lo cual repercute, entre otros aspectos, en la propia tarea deportiva, en el número de actividades y en el sistema de competición, así como también en el papel de los principales actores. El entrenador, junto con el deportista y los gestores deportivos, juegan un papel fundamental en el proceso inclusivo que supone toda práctica deportiva (Segura, Martínez-Ferrer y Guerra, 2011) y en el caso del deporte adaptado, la importancia se incrementa.

El técnico de deporte adaptado, además de los conocimientos habituales para dirigir un grupo deportivo, debe atender otros aspectos como el tipo de discapacidad, las necesidades específicas del deportista, las mayores diferencias individuales, la influencia del contexto en los diversos niveles de aprendizaje, los sistemas especiales de entrenamiento y competición, o los aspectos del contexto familiar y social (Martínez-Ferrer, 2008). Un buen entrenador de deporte adaptado debe afrontar situaciones más complejas que las habituales y conocer las limitaciones y los puntos fuertes de sus deportistas. Por tanto, para el buen ejercicio de su rol, el téc- nico debe desarrollar habilidades más específicas, que Sanz lo focaliza en la conducta del entrenador durante el proceso de entrenamiento y en la formación del técnico de deporte adaptado (Sanz, 2003, 2009).

Los avances experimentados por el deporte adaptado ponen en evidencia una mayor necesidad de profesionalización de los técnicos, que pasa por una mejor especialización en los aspectos relacionados con la dirección del entrenamiento, los hábitos y el estilo de vida de los deportistas, en función de los diversos tipos de discapacidad, el conocimiento de aspectos específicos de la iniciación deportiva, los sistemas de competición y los aspectos médicos y psicológicos.

El desarrollo del deporte adaptado sigue el curso de las transformaciones sociales. Desde el punto de vista de la organización deportiva, hablar de un técnico es hacerlo en tanto que líder que ejerce un rol prescrito desde el sistema socio-deportivo y desde las expectativas del propio grupo. Es un rol doblemente funcional: técnico (centrado en la tarea) y socio-educativo (centrado en el desarrollo como persona y en objetivos psicológicos no necesariamente relacionados con la excelencia en el rendimiento) (Segura, 1990). En el deporte, el liderazgo afronta la doble exigencia de ocuparse de la vertiente personal y de la vertiente social del deportista, ya que facilita su desarrollo personal, a la vez que su rendimiento sufre una exigencia social por los resultados. Dick (1989) afirma que, para ser completamente efectivo en el arte del entrenamiento, el entrenador debe comprender claramente la intención de cada práctica y su relevancia en el esquema total de preparación y, al mismo tiempo, comprender la transformación, el cambio personal del atleta y el rol del deporte de su vida. El técnico se preocupa de adaptar las actividades a las necesidades y posibilidades del deportista y de lograr un estado físico y psíquico óptimo. La doble funcionalidad es una tensión presente en toda práctica deportiva, que en el deporte adaptado adquiere un relieve singular, que se concreta en la dialéctica entre objetivos de rendimiento y objetivos personales, y el interés por la persona y su bienestar (Lisbona, 2006). En esta línea, Pérez-Ramírez (2002a) habla del significado de la "eficacia" del entrenador y destaca que éste debe poseer habilidades para actuar en relación a las características y necesidades de los deportistas.

Existen estudios que investigan otros aspectos relacionados con el ejercicio del rol de entrenador. Por ejemplo, Leo-Marcos, Sánchez, Sánchez, Amado y Garcia (2009) se centran en el compromiso deportivo del entrenador y su influencia en el clima motivacional (Balaguer, Castillo, Álvarez y Duda, 2009) en la relación entre la percepción de apoyo a la autonomía del entrenador, la satisfacción de sus necesidades psicológicas y en cómo influye en los deportistas.

Algunos especialistas han investigado sobre la importancia de la formación del entrenador. Unos destacan como primera necesidad la formación de los aspectos técnicos (Jiménez, Lo- 
renzo, Borrás, Gómez y Física, 2009). Otros opinan que el técnico de deporte adaptado necesita ir más allá de las especificaciones técnico-tácticas y debe ser experto en las características específicas de los deportistas, en función del tipo de discapacidad, además de desarrollar otras competencias, como las de gestión y comunicación social (DePauw y Doll-Tepper, 1989).

La perspectiva de la Psicología Positiva complementa conocimientos de la psicología tradicional, que según algunos expertos constituye un nuevo paradigma que tiene como constructos el optimismo, la felicidad, la autoestima y el Engagement (Hervás, 2009) y que puede ser muy útil para estudiar el rol del técnico deportivo, en concreto del técnico que trabaja en deporte adaptado.

El Engagement es un constructo complementario al Burnout y surge de él (Maslach, Schaufeli, y Leiter, 2001). Durante mucho tiempo, la psicología social y de las organizaciones ha estudiado el fenómeno del Burnout en el desempeño de roles. En el ámbito de la Psicología del Deporte hay literatura acerca del Burnout en técnicos deportivos (Carlin y De los Fayos, 2010), pero muy poca que trate el Engagement. Freudenberger (1974) introdujo el constructo Burnout definiéndolo como pérdida de energía, falta de motivación y falta de interés por todo lo relacionado con el trabajo, hasta llegar al gran agotamiento. Maslach y Jackson (1996) entienden que el Burnout engloba tres síntomas: cansancio emocional, despersonalización/cinismo y sentimientos de poca eficacia profesional. Por otro lado, Schaufeli y Enzmann (1998) lo describen como un estado mental persistente, negativo, relacionado con el trabajo del individuo caracterizado por agotamiento emocional, que se acompaña de malestar, de una motivación reducida y del desarrollo de actitudes disfuncionales hacia la tarea.

El Engagementse se define como un estado mental positivo relacionado con el trabajo, afectivo-cognitivo persistente, que no está focalizado en un objeto, evento o situación particular (Schaufeli, Salanova, González-Roma y Bakker, 2002; Salanova y Schaufeli, 2004). Como sucede con el Burnout, se le conciben tres dimensiones: vigor, dedicación y absorción. El vigor se refiere al ámbito laboral y se caracteriza por altos niveles de energía y de resistencia mental, persistente en las situaciones y momentos dificultosos; la dedicación, a la implicación laboral de la persona, con sentimientos de significación y de entusiasmo, inspiración, orgullo y reto por su trabajo; y la absorción, a la concentración en el trabajo, que permite a la persona sentir que el tiempo pasa rápido y que disfruta de sus tareas (Schaufeli et. al, 2002).

Así como se ha demostrado que el Burnout puede provocar bajo rendimiento e incluso abandono del trabajo, el enfoque del Engagement concibe un desarrollo positivo en el ejercicio del rol de técnico y de prevención del desgaste y el abandono. Si se conoce el funcionamiento del Engagement se logrará un mejor desempeño del rol y se orientarán mejor los procesos de formación y de promoción de competencias importantes del técnico, así como el incremento de la satisfacción, la mejora de los índices de salud, la consolidación de una buena carrera profesional y la reducción del abandono.

El objetivo principal de este estudio es realizar un análisis descriptivo de la trayectoria del técnico de deporte adaptado en el ámbito nacional y conocer su Engagement, comparándolo con los técnicos de deporte general. Otro objetivo es comprobar si hay una relación entre las variables autovaloración, satisfacción personal, motivación personal y rendimiento de los deportistas, así como la relación de estas variables con el nivel de Engagement, en sus tres dimensiones de Vigor, Dedicación y Absorción.

Las hipótesis planteadas en este estudio son: 1. A mayor puntuación en autovaloración, satisfacción personal, motivación personal y rendimiento de sus deportistas, el técnico presenta más Engagement, en el nivel general y en cada una de sus dimensiones; 2. A más edad del técnico, más nivel de Engagement; y 3. El técnico de deporte adaptado presenta un nivel de Engagement superior al técnico de deporte general.

\section{Método}

\section{Participantes}

La muestra de esta investigación ha sido seleccionada de forma no probabilística e intencional. Está formada por 90 participantes de entre 24 y 61 años, divididos en 45 técnicos de deporte adaptado (32 hombres - 13 mujeres) (edad: 38.99 años \pm 10.14$)$ y 45 técnicos de deporte general (32 hombres 13 mujeres) (edad: 32.33 ańos \pm 12.02 ), de Cataluña y Madrid.

Para pertenecer al grupo de estudio fue necesario cumplir los siguientes criterios de inclusión: tener como mínimo 1 año de experiencia y trabajar como técnico al menos 3 horas semanales. En el momento de responder a los cuestionarios, los técnicos de deporte adaptado debían estar en activo, con contrato laboral, entrenando deportistas con discapacidad que estuvieran participando en competiciones oficiales. Los técnicos entrenaban a deportistas que practicaban Baloncesto, Atletismo, Natación, Futbol, Tenis de Mesa, Tenis, Hípica, Esquí y Voleibol. Como grupo control, participaron técnicos de deporte general vinculados a los mismos deportes que, cumpliendo los mismos criterios de inclusión, éstos no entrenaban deportistas con discapacidad.

\section{Instrumentos}

Para la realización de este estudio se han utilizado dos instrumentos: un breve cuestionario elaborado "ad hoc"sobre la trayectoria profesional del técnico y el cuestionario validadoUWES -Utrecht WorkEngagementScale (Schaufeli y Bakker, 2003), que evalúa el nivel de Engagement con sus tres dimensiones (vigor, dedicación y absorción), en su versión española adaptada 
(Benevides-Pereira, Fraiz de Camargo y Ponto-Camargo, 2009).

El cuestionario sobre la trayectoria profesional de técnicos-entrenadores de deporte, se ha elaborado específicamente para este trabajo con la finalidad de recoger información sobre las características de la trayectoria profesional de los técnicos, basado inicialmente en las variables del estudio de Pérez-Ramírez (2002b). Se elaboró una primera versión del cuestionario, que fue sometida al juicio de 3 expertos en el ámbito de la actividad física adaptada, investigadores acreditados de dos grupos de investigación del ámbito del deporte y del ámbito de la Psicología de la Universitat Ramon Llull. La segunda versión, con las sugerencias aportadas por los expertos, fue dada a responder a cuatro técnicos deportivos, tanto de deporte adaptado como general, y contando con sus comentarios se redactó la versión definitiva. El cuestionario ha facilitado la obtención de conocimiento sobre variables agrupadas bajo el título de trayectoria profesional e identidad: datos personales, contexto profesional, estudios realizados, otras experiencias laborales (no vinculadas a la práctica como entrenador), influencias para la práctica deportiva, experiencia como entrenador y auto percepción como entrenador.

El cuestionario UWES consta de 17 ítems referidos a tres escalas: Vigor (6 ítems), la Dedicación (5 ítems) y a la Absorción (6 ítems). Cada ítem puede ser puntuado entre 0 y 6 . El tiempo de aplicación es de 10 minutos aproximadamente, y se puede realizar tanto individual como colectivamente.

Los resultados del cuestionario se pueden obtener escala por escala, y posteriormente el sumatorio de las tres escalas para obtener el nivel general de Engagement del participante. La puntuación máxima a conseguir en cada dimensión y en el global son 6 puntos.

En la muestra utilizada el cuestionario tiene un coeficiente de alfa de Cronbach de 0.871 , y en cada una de sus dimensiones: Vigor de 0.762 , Dedicación de 0.833 y Absorción de 0.857 .

\section{Procedimiento}

Para realizar el estudio se contactó con federaciones de deporte adaptado y clubes deportivos; se les explicaron los objetivos del estudio y se solicitó su colaboración en la pre-selección de los participantes. Las organizaciones colaboradoras representan los diferentes ámbitos del deporte adaptado: discapacidad física, discapacidad sensorial y discapacidad intelectual. Posteriormente, se realizaron las gestiones con federaciones y clubes deportivos de deporte general equivalentes. A los técnicos pre-seleccionados por estas organizaciones, que cumplían los criterios de inclusión, se les explicaron los objetivos del estudio y se les invitó a participar de forma anónima y confidencial.

\section{Análisis de los datos}

Una vez recogidos y analizados los cuestionarios cumplimentados, se procedió a la introducción de los datos en la aplicación informática y al análisis estadístico de los resultados, mediante el software StatisticalPackagefor Social Sciences (version 18.0 para Windows, SPSS Inc, Chicago, IL, USA).

Se realizó un análisis descriptivo de las principales variables incluyendo: media, moda, frecuencias, desviación típica. Posteriormente, la prueba Kolmogorov-Smirnov para comprobar si las variables de la muestra se ajustaban a la normalidad. Para observar la relación entre las variables del grupo focal (técnicos de deporte adaptado) se usó el procedimiento de correlaciones bivariadas, concretamente la correlación de Spearmanpara observar si existían correlaciones significativas entre las variables. Para comparar las medias de una misma variable para dos grupos diferentes (técnicos de deporte adaptado y técnicos de deporte general) y para ver si existían diferencias entre géneros se aplicó la prueba $T$ para muestras independientes, en aquellas variables en que la prueba de normalidad se ajustaba a la normal, y la prueba U de Mann Whitney en los casos que no se ajustaban a la normal. El nivel de significación se estableció a $\mathrm{p}<0.05$.

\section{Resultados}

Presentamos en primer lugar el análisis descriptivo de las variables estudiadas, en relación a los técnicos de deporte adaptado y los técnicos de deporte general (tabla 1).

Tabla 1. Análisis de frecuencias de los técnicos de deporte adaptado y general.

\begin{tabular}{|c|c|c|c|c|c|c|}
\hline & \multicolumn{3}{|c|}{ Técnicos de deporte adaptado } & \multicolumn{3}{|c|}{ Técnicos de deporte general } \\
\hline & Media & Moda & Desv.Típ. & Media & Moda & Desv.Típ. \\
\hline Edad & 38.33 & 26 & 10.14 & 32.33 & 20 & 12.06 \\
\hline Años de experiencia & 13.76 & 10 & 9.07 & 12.04 & 2 & 11.51 \\
\hline Número de horas-semana & 21.6 & 40 & 14.20 & 16.47 & 6 & 9.99 \\
\hline Autovaloración como técnico (0-5) & 3.98 & 4 & 0.67 & 3.8 & 3 & 0.80 \\
\hline Nivel satisfacción personal (0-5) & 4.11 & 4 & 0.87 & 4.13 & 4 & 0.76 \\
\hline Nivel de motivación personal (0-5) & 4.6 & 5 & 0.70 & 4.53 & 5 & 0.69 \\
\hline
\end{tabular}




\begin{tabular}{rcccccc}
\hline & \multicolumn{2}{c}{ Técnicos de deporte adaptado } & \multicolumn{3}{c}{ Técnicos de deporte general } \\
\hline & Media & Moda & Desv.Típ. & Media & Moda & Desv.Típ. \\
\hline Rendimiento de sus deportistas (0-5) & 3.95 & 4 & 0.91 & 3.71 & 4 & 0.66 \\
Vigor (UWES) & 51.38 & 5.5 & 0.75 & 48.48 & 4.66 & 0.78 \\
Dedicación (UWES) & 52.93 & 5.6 & 0.64 & 50.67 & 4.2 & 0.73 \\
Absorción(UWES) & 45.56 & 4.83 & 0.81 & 45.81 & 4.33 & 0.83 \\
Nivel de Engagement & 49.33 & 4.94 & 0.67 & 48.16 & 4.05 & 0.71 \\
\hline
\end{tabular}

Según el análisis de frecuencias del grupo focal, se observa

deporte adaptado (Tabla 2). cuál es el perfil de la trayectoria profesional de los técnicos de

Tabla 2. Perfil de la trayectoria de los técnicos de deporte adaptado

\begin{tabular}{|c|c|c|}
\hline \multicolumn{3}{|c|}{ Perfil de Formación } \\
\hline Nivel de estudios & & Influencias de vinculación \\
\hline 40\% Licenciado & 46.7\% Deporte & $6.7 \%$ Otros \\
\hline $26.7 \%$ Otros & 20\% Familia & 6.7\% Diversas \\
\hline $22.2 \%$ Máster & $11.1 \%$ Amigos & $2.2 \%$ Cultura \\
\hline $8.9 \%$ Bachillerato & $6.7 \%$ Escuela & \\
\hline $2.2 \%$ Graduado escolar & & \\
\hline \multicolumn{3}{|c|}{ Trayectoria del Técnico } \\
\hline Experiencia previa como deportista & Tipo de deporte & Exclusividad de trabajo \\
\hline $37.8 \%$ Competición & $37.8 \%$ Más de un deporte & 68.9\% No Exclusivamente \\
\hline $22.2 \%$ Semiprofesional o profesional & $17.8 \%$ Baloncesto & 31.1\% Exclusivamente \\
\hline $20 \%$ Alta competición & $13.3 \%$ Otros & \\
\hline 17.8\% Iniciación & $11.1 \%$ Natación & \\
\hline \multirow[t]{3}{*}{$2.2 \%$ Tecnificación } & $8.9 \%$ Atletismo & \\
\hline & 6.7\% Tenis de Mesa & \\
\hline & 4.4\% Futbol & \\
\hline
\end{tabular}

Una vez se realizaron las pruebas de normalidad (Prueba de Kolmogorov-Smirnov), se analizó el procedimiento de correlaciones bivariadas, concretamente la correlación de Spear- man, con objeto de comprobar cómo están relacionadas la variables de los técnicos de deporte adaptado (Tabla 3).

Tabla 3. Correlaciones bivariadas (Spearman), de los técnicos de deporte adaptado.

\begin{tabular}{|c|c|c|c|c|c|c|c|c|c|c|c|}
\hline Variables & 1 & 2 & 3 & 4 & 5 & 6 & 7 & 8 & 9 & 10 & 11 \\
\hline 1.-Edad & - & $.761^{* *}$ & & $.395^{* *}$ & & & & & & & \\
\hline 2.-Años experiencia & & - & $.445^{* *}$ & $.512^{* *}$ & $.322^{*}$ & & & & & & \\
\hline 3.-Número de horas & & & - & $.454^{* *}$ & & & $-.330^{*}$ & & & & \\
\hline 4.-Autovaloración & & & & - & $.460^{* *}$ & & & & & & \\
\hline 5.-Nivel satisfacción & & & & & - & $.413^{* *}$ & & $.459^{* *}$ & $.536^{*}$ & $.358^{*}$ & $.518^{* *}$ \\
\hline 6.-Nivel motivación & & & & & & - & & & $.372^{*}$ & & \\
\hline 7.-Rendimiento de susdeportistas & & & & & & & - & & & & \\
\hline 8.-Vigor (UWES) & & & & & & & & - & $.618^{* *}$ & $.604^{* *}$ & $.758^{* *}$ \\
\hline 9.-Dedicación (UWES) & & & & & & & & & - & $.612^{* *}$ & $.787^{* *}$ \\
\hline 10.-Absorción (UWES) & & & & & & & & & & - & $.826^{*}$ \\
\hline 11.- NivelEngagement & & & & & & & & & & & - \\
\hline
\end{tabular}


Para conocer si existen diferencias entre los técnicos de deporte adaptado y los técnicos de deporte general, se realizaron pruebas de comparación de medias para muestras independientes. En el caso de Vigor, Dedicación, Absorción y Nivel de Engagement se ha aplicado la prueba T de Student para muestras independientes, ya que se ajustan a la normalidad.

En la prueba $T$ para muestras independientes para observar la diferencia entre los dos grupos (técnicos de deporte adaptado y técnicos de deporte general) no existen diferencias significativas en ninguna de las dimensiones ( $p>0.05)$ : Vigor $(\mathrm{p}=0.076)$, Dedicación $(\mathrm{p}=0.122)$, Absorción $(\mathrm{p}=0.886)$ y Nivel de Engagement $(\mathrm{p}=0.425)$. Posteriormente se ha realizado la prueba $T$ para muestras independientes de la variable género, en cada uno de los grupos. En el grupo de los técnicos de deporte adaptado no se observan diferencias significativas $(p>0.05)$ : Nivel de Engagement $(p=0.99)$, Vigor $(p=0.66)$, Dedicación $(\mathrm{p}=0.68)$, Absorción $(\mathrm{p}=0.47)$. En el grupo de los técnicos de deporte general tampoco se observan diferencias entre géneros en las variables estudiadas ( $p>0,05)$ : Nivel de Engagement $(\mathrm{p}=0.96)$, Vigor $(\mathrm{p}=0.98)$, Dedicación $(\mathrm{p}=$ $0.88)$ y Absorción $(\mathrm{p}=0.82)$.

En el caso de las variables que no se ajustan a los criterios de normalidad se utilizó una prueba no-paramétrica, la $U$ de Mann Whitney para variables independientes, para la comparación de las medias. Se observa que en todos los casos no hay diferencias entre grupos ( $p>0.05)$ : autovaloración como técnico ( $\mathrm{p}=0.199)$, nivel de satisfacción personal $(\mathrm{p}=0.889)$, nivel de motivación personal $(\mathrm{p}=0.603)$ y rendimiento de sus deportistas $(0,078)$.

Finalmente se realizó la U de Mann Whitney, para observar si existen diferencias entre géneros, en cada uno de los grupos, no observándose diferencias en ninguno de los casos (Tabla5). En los técnicos de deporte adaptado ( $>$ > 0.05): Autovaloración como técnico $(\mathrm{p}=0.18)$, Nivel de satisfacción $(\mathrm{p}=0.47)$, Nivel de motivación $(\mathrm{p}=0.20)$ y Rendimiento de los deportista $(\mathrm{p}=0.36)$. Tampoco se observan diferencias significativos entre generos en el grupo de técnicos de deporte general ( $p>0.05)$ : Autovaloración como técnico ( $p=0.45)$, Nivel de satisfacción $(\mathrm{p}=0.60)$, Nivel de motivación $(\mathrm{p}=$ $0.28)$ y Rendimiento de los deportista $(\mathrm{p}=0.70)$.

\section{Discusión}

Los resultados muestran características del grupo de técnicos de deporte adaptado, los cuales poseen un nivel de estudios alto: un $40 \%$ son licenciados y un $22,2 \%$ han cursado estudios de máster (Tabla 2). La experiencia del técnico es una variable importante, porque además de dotar conocimiento, otorga confianza en las propias competencias. Hay relación positiva entre la experiencia, el nivel de autovaloración y el de satisfacción del técnico de deporte adaptado. La formación del técnico es uno de los puntos clave para poder realizar sus funciones de manera óptima y conseguir un buen rendimiento de los deportistas, tal y como afirman Moreno y Del Villar (2004, citados en Jiménez et. al., 2005; Sanz, 2003).

Para conocer las principales influencias de vinculación a la práctica deportiva en los técnicos de deporte adaptado, se propusieron las opciones: deporte, familia, amigos, escuela, cultura, otros o diversas. Los técnicos consideran que la mayor influencia procede de la práctica deportiva en sí misma $(46,70 \%)$, seguida de la influencia familiar (20\%) (Tabla 3). Se considera la influencia familiar como un elemento facilitador para la práctica deportiva, y en este sentido Jaarsma, Dijkstra, Geertzen y Dekker (2014) afirman que un factor ambiental puede ser una barrera para el deporte, mientras que los factores personales son facilitadores para la práctica deportiva.

El 80\% de la muestra de técnicos de deporte adaptado han participado en el mundo de la competición como deportistas. De ellos, el $20 \%$ en la alta competición y el $22,20 \%$ como deportistas semi-profesionales o profesionales (Tabla 2). Así pues, haberse vinculado a la competición como deportista puede concebirse como un estímulo facilitador para un mejor desempeño del rol de técnico. Muchos técnicos han tenido una larga trayectoria como deportistas y se puede considerar un indicador de que la profesión de técnico podría ser una continuación de carrera en el ámbito del deporte, desde la práctica como deportista.

En cuanto al compromiso con la actividad, más del $60 \%$ de los técnicos de deporte adaptado no se dedican exclusivamente a esta tarea (Tabla 2). Las observaciones dadas por los participantes muestran dos perfiles. De una parte, hay técnicos que a pesar de no ejercer exclusivamente como tales y desempeñar una segunda profesión próxima al ámbito deportivo o educativo: docencia (educación física en escuelas), gestión deportiva, o combinación de la carrera de deportista con la de técnico. Por otro lado, también hay técnicos que combinan las funciones de técnico deportivo con otra actividad profesional no vinculada al mundo del deporte. Las razones podrían estar relacionadas con las causas de subsistencia económica (como varios técnicos manifiestan en el apartado de observaciones), que es una barrera para ejercer esta tarea, lo que coincide también en el campo del deporte general en los resultados del estudio realizado por Pérez-Ramírez (2002b). Muchos técnicos de deporte adaptado no pueden dedicarse exclusivamente a este trabajo, por lo quesería interesante ampliar el estudio para investigar datos sobre el abandono y sus causas, dado que la muestra estudiada presenta elevadas puntuaciones de Engagement (media de 4,93 y algún participante incluso la puntuación máxima de 6), que puede interpretarse como un factor importante de motivación por la permanencia en el rol y de prevención del abandono.

Respecto al tipo de deporte adaptado, es la única variable de la trayectoria profesional en la que se observan diferencias 
entre el grupo de técnicos de deporte adaptado y el grupo de técnicos de deporte general. El 37,80\% de los técnicos de deporte adaptado se dedican a entrenar más de un tipo de deporte, a diferencia de los técnicos de deporte general $(11,70 \%)$. Se explicaría porque en el deporte en general se tiende a la especialización en un tipo de deporte, sobre todo a medida que se prospera en el nivel de competición. Sin embargo, en las federaciones y clubes de deporte adaptado, que son polideportivos, es frecuente que un deportista participe en competiciones de más de un deporte, no sólo en el período de iniciación, sino también en las etapas de tecnificación y de competición. Así pues, en base a estos resultados, se aprecia una tendencia polideportiva en los técnicos de deporte adaptado.

El 24\% de los técnicos de deporte general afirman haber estado vinculados de manera puntual al deporte adaptado. Las razones manifestadas por algunos de los participantes son reveladoras: "Es muy gratificante, es gente que confía en ti totalmente" o "Es muy beneficioso e instructivo". En todos los casos, la experiencia ha sido positiva y muchos afirman que la causa de no haber continuado vinculados a este ámbito ha sido la falta de oportunidad o porque en su club la inclusión de personas con discapacidad es aislada y discontinua: "Ahora ya no hay nadie", "Se apuntó en el club un jugador con una deformación en la mano, que participaba en torneos de discapacidad", "Cuando estudiaba en la universidad dí gimnasia a una persona con discapacidad visual".

La satisfacción del técnico de deporte adaptado es un elemento clave en su autovaloración general de rol y tiene relación con la motivación y con el nivel de Engagement. Los resultados están en línea con las opiniones de Salanova et. al. (2004), en cuanto que los empleados con un nivel más elevado de Engagement se sienten más satisfechos con el trabajo, mantienen una actitud más activa y dinámica hacia él y les fomenta mayor motivación.

Podemos suponer que las observaciones de Salanova y et. al., (2000), en cuanto a que el Engagement es fundamental para el bienestar psicológico del trabajador, se dan en el técnico deportivo adaptado. En esta línea, hay correlación significativa con las variables que hacen referencia a la percepción del técnico respecto a satisfacción, motivación, autovaloración general como técnico y rendimiento de sus deportistas. Destaca la correlación positiva entre el nivel de satisfacción y las tres dimensiones específicas del Engagement, así como con su nivel general. También, entre nivel de motivación y la dedicación. Es evidente que los técnicos de deporte adaptado experimentan un buen desempeńo del rol y una buena carrera como tal, ya sea a dedicación plena o parcial.

En el caso de los técnicos de deporte adaptado no hay relación significativa entre la edad y el nivel de Engagement, a diferencia del estudio de Schaufelli y Bakker (2004), que halló correlación positiva y significativa entre la edad del traba- jador y el Engagement. Se podría hipotetizar que la causa es que gran parte de los participantes no ejercen exclusivamente el rol de técnico de deporte o que el ejercicio de técnico de deporte provee especial desarrollo personal. En este sentido, entre las tres dimensiones de Engagement (Vigor, Dedicación y Absorción) hay correlación positiva, coincidiendo con los estudios de Salanova y Shaufeli (2004).

En las variables del Engagement no hay diferencia entre los técnicos de deporte adaptado y los técnicos de deporte general. Los resultados ponen en evidencia la semejanza entre ambos grupos, en cuanto a esas variables, y se confirma que la mera implicación de los actores en los roles de técnico deportivo es de por sí gratificante y motivadora, ejerzan con grupos de deportistas con o sin discapacidad. La participación en el "rol de técnico" es potenciadora de Engagement, en ambos grupos de deportistas. Sin embargo, cabe destacar el elevado número de técnicos de deporte general que a lo largo de su carrera han estado vinculados al deporte adaptado y han entrenado deportistas con discapacidad, y han opinado de forma afirmativa sobre cómo continuar entrenando deportistas con estas características. Podemos hipotetizar que la desvinculación con el grupo de deporte adaptado fue debida casi siempre a variables relacionadas con la organización y se propone profundizar en el estudio de las causas, introduciendo el constructo de Commitment, esto es, del compromiso con la organización.

\section{Conclusiones}

No aparecen diferenciasen las dimensiones de Engagement de los dos grupos estudiados (técnicos de deporte adaptado y técnicos de deporte general). Los técnicos de deporte adaptado que han participado se caracterizan por tener un nivel de estudios universitarios (62.2\%). Su principal influencia para vincularse a la práctica deportiva es "el deporte en sí mismo" (46.7\%). El técnico de deporte adaptado tiene un perfil más polideportivo (37.8\%) que el resto. Gran parte de los técnicos de deporte adaptado no se dedican exclusivamente a esta tarea (68.9\%). Los técnicos de deporte adaptado inciden en las dificultades de poder ejercer esta profesión como su único trabajo (debido al salario).

En cuanto a las variables de auto-percepción; hay relación entre la autovaloración y el nivel de satisfacción, y entre el nivel de satisfacción y el nivel de motivación. Por lo tanto, cuanto más se autovaloran, los técnicos más satisfechos están. Además hay correlación entre su nivel de motivación y la dimensión de Dedicación de la escala UWES. También, cuanto más nivel de satisfacción personal experimentan, mayor y más completo es su nivel de Engagement, en las tres dimensiones de Vigor, Dedicación y Absorción. En cuanto al género no se han encontrado diferencias significativas.

El estudio presenta limitaciones en cuanto a la muestra. 
Sería conveniente incrementar el número de mujeres participantes, así como el número de modalidades deportivas de los técnicos. Ese incremento permitiría, como línea de futuro, diferenciar entre el tipo de discapacidad, física, psíquica o sensorial, de los deportistas entrenados por lo técnicos. A su vez, se podría ampliar a un tercer grupo en el estudio, formado por técnicos que trabajan de forma inclusiva, entrenando deportistas sin y con discapacidad. También, en estudios posteriores, se podría realizar un estudio de contenido de las respuestas dadas por los participantes en el apartado de observaciones, que permitiera analizar comentarios relacionados con su experiencia en deporte adaptado y en la relación a los deportistas con discapacidad.

\section{Aplicaciones prácticas}

Este estudio ha sido una primera aproximación al estudio psicosocial en el ámbito de los técnicos de deporte adaptado. Los resultados proporcionan evidencias acerca de la experiencia que muchos técnicos han podido tener con deportistas con discapacidad, así como la actitud positiva de muchos técnicos de deporte general a entrenar deportistas con discapacidad. Estas evidencias nos sugieren que la investigación futura sobre el rol de los técnicos de deporte adaptado puede integrarse en el ámbito del deporte general, lo que significaría un paso adelante en la normalización en el deporte. Además, este estudio aporta a los clubes y federaciones información sobre el perfil de los técnicos que trabajan con deportistas con discapacidad; también, sugerencias para mejorar aspectos como la contratación e inclusión de técnicos en los clubs y sus planes de formación y reciclaje. Ya a nivel más científico, se aporta conocimiento sobre el constructo de Engagement, que implica la novedad del enfoque de la psicología positiva en este ámbito, abriendo una nueva línea de investigación.

Financiación de la investigación: Comité Paralímpico Español y Consejo Superior de Deportes (Proyecto 021/ UPR10/12).

Agradecimientos: Federació Catalana d'Esports de Persones amb Discapacitat Física, Comité Paralímpico Español y Consejo Superior de Deportes.

\section{Referencias}

1. Balaguer, I., Castillo, I., Álvarez, M. S. y Duda, J. L. (2009). Coach autonomy support and quality of sport engagement in young soccer players. The Spanish Journal of Psychology, 12(1), 138-148.

2. Benevides-Pereira, A.M., Fraiz de Camargo, D. y Ponto-Camargo, P.C. (2009). Escala Utrecht de Engagement en el trabajo. Occupational Health Psychology Unit Ultrecht University.

3. Carlin, M. y de los Fayos, E. J. G. (2010). El síndrome de burnout: Evolución histórica desde el contexto laboral al ámbito deportivo. Anales de Psicología, 26 (1), 169-180.

4. Consejo Superior de Deportes. (2009). Plan integral para la actividad física, CSD versión1. Madrid: CSD.

5. DePauw K.P. y Doll-Tepper, G. (1989). European perspectives on Adapted Physical Activity. Adapted Physical Activity Quarterly, 6, 9599.

6. Dick, F.W. (1989). Guide lines for coaching conduct. Athletics Coach, 23, 3-6.

7. Freudenberger, H. J. (1974). Staff burnout. Journal of social issues, 30(1), 159-165.

8. Guttmann, L. (1976). Textbook of sport for the disabled. Brisbane: University of Queensland Press.

9. Hervás, G. (2009). Psicología positiva: Una introducción. Revista Interuniversitaria de Formación del Profesorado, 23(66), 23-41.

10. Jaarsma, E. A., Dijkstra, P. U., Geertzen, J. H., y Dekker, R. (2014). Barriers to and facilitators of sports participation for people with physical disabilities: A systematic review. Adapted Physical Activity Quarterly, 31 (3), 240-264.

11. Jiménez, S., Lorenzo, A., Borrás, P. J. y Gómez, M. A. (2009) El compromiso deportivo en entrenadores de baloncesto de alto rendimiento. Cuadernos de Psicología del Deporte, 9, 25.

12. Leo-Marcos, F., Sánchez, P., Sánchez, D., Amado, D. y García, T. (2009). Influencia del clima motivacional creado por el entrenador en el compromiso deportivo en jóvenes jugadores de baloncesto. Cuadernos de Psicología del Deporte, 9, 49-57.

13. Lisbona, A. (2006). Hacia un modelo en la práctica del deporte adap- tado (deporte adaptado e integración). Revista de Educación Social, 5, 15-18.

14. Marslach, C., Jackson, S. E. y Leiter, M. (1996). Maslach Burnout Inventory, Test manual. Palo Alto, CA: Consulting Psychologists Press.

15. Marslach, C., Schaufeli, W. B. y Leiter, M.P. (2001). Job Burnout. Annual Review of Psychology, 52, 397-422.

16. Martínez-Ferrer, J. O. (2008). Paper de l'activitat física i l'esportadaptat en la normalització de les persones discapacitades. Aloma: Revista de Psicologia, Ciènciesde l'Educació i de l'Esport Blanquerna, 13, 13-23.

17. Pérez-Ramírez, M. C. (2002a). Caracterización del entrenador de alto rendimiento deportivo. Cuadernos de Psicologia del Deporte, 2(1), 16-37.

18. Pérez-Ramírez, M. C. (2002b). Estudio cualitativo sobre entrenadores de alto rendimiento deportivo. Revista de Psicología del Deporte, 11(1), 9-33.

19. Pérez-Tejero, J. (2009). La investigación en actividades físicas y deportes adaptados: Un camino aún por recorrer. RICYDE. Revista Internacional de Ciencias Del Deporte, 5(16), 1-3.

20. Reina, R. (2014). Adapted Physical Activity: the journey to Ithaca goes ahead. RICYDE. International Journal of Sport Science, 37, 177-179

21. Salanova, M. y Schaufeli, W. (2004). La ilusión por el trabajo (engagement): ¿el lado positivo del burnout. Revista de Psicología del Trabajo y las Organizaciones, 11, 73-92.

22. Salanova, M., Schaufeli, W. B., Llorens, S., Peiro, J. M. y Grau, R. (2000). Desde el 'burnout'al 'engagement': juna nueva perspectiva. Revista de Psicología del Trabajo y las Organizaciones, 16(2), 117-134.

23. Sanz, D. (2003). Análisis y optimización de la conducta del entrenador de tenis en silla de ruedas de alta competición durante el proceso de entrenamiento. Tesis doctoral inédita: Universidad Extremadura.

24. Sanz, D. (2009). La formación de técnico en el deporte adaptado y su inclusión en la formación deportiva general. En Pérez, J. y Sanz, D. (eds.) Libro de actas de la $1{ }^{\text {a }}$ Conferencia Nacional de Deporte Adaptado. Toledo: Consejo superior de Deportes y Comité Paralímpico Español.

25. Segura, J. (1990). L'entrenadoresportiu: anàlisid'un rol. Tesis doctoral no publicada. Universitat de Barcelona. 
26. Segura, J.S., Martínez-Ferrer, J. O. y Guerra, M., (2011). FocusGroup: Una metodología para fomentar procesos de inclusión en el deporte. Comunicación presentada a $3^{\text {a }}$ Conferencia Nacional de Deporte Adaptado. Valencia. España.

27. Seligman, M. E. P., Steen, T. A., Park, N. y Peterson, C. (2005). Positive psychology progress: Empirical validation of interventions. American Psychologist, 60(5), 410-421.

28. Schaufeli, W. yBakker, A. (2003). Escala Utrecht de Engagement en el Trabajo. Valencia:Utrecht University.
29. Schaufeli, W.B. y Enzmann, D. (1998). The burnout companion to study and research. A critical analysis. London: Taylor

30. Schaufeli, W., Salanova, M., Gonzalez-Roma, V. y Bakker, A. B. (2002). The measurement of engagement and burnout: A two sample confirmatory factor analytic approach. TheJournal of HappinessStudies, 3,71-92.

31. Vera, B. (2006). Psicología positiva: Una nueva forma de entender la psicología. Papeles del Psicólogo, 27(1), 3-8. 